\section{PSICOLOGÍA IBEROAMERICANA}

\section{Psicología lberoamericana}

ISSN: 1405-0943

psicología.iberoamericana@uia.mx

Universidad Iberoamericana, Ciudad de

México

México

Hernández Montoya, Dewi Sharon; Benjet, Corina; Nishimura Meguro, Elisa; Hernández Méndez, María Elena

Validez y confiabilidad del Cuestionario de Representación de la Enfermedad para Diabetes (CRED) en adolescentes mexicanos

Psicología Iberoamericana, vol. 20, núm. 1, enero-junio, 2012, pp. 40-47

Universidad Iberoamericana, Ciudad de México

Distrito Federal, México

Disponible en: http://www.redalyc.org/articulo.oa?id=133924623005

Cómo citar el artículo

- Número completo

- Más información del artículo

- Página de la revista en redalyc.org

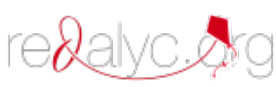

Sistema de Información Científica

Red de Revistas Científicas de América Latina, el Caribe, España y Portugal Proyecto académico sin fines de lucro, desarrollado bajo la iniciativa de acceso abierto 


\title{
Validez y confiabilidad del Cuestionario de Representación de la Enfermedad para Diabetes (CRED) en adolescentes mexicanos
}

\author{
Validity and Reliability of the IIIness Representation Questionnaire (DIRQ) \\ in Mexican Adolescents
}

\author{
Dewi Sharon Hernández Montoya* \\ Universidad Nacional Autónoma de México, México \\ Corina Benjet \\ instituto Nacional de Psiquiatría Ramón de la Fuente Muñiz, México \\ Elisa Nishimura Meguro, María Elena Hernández Méndez \\ Hospital de Pediatría. Centro Médico Nacional S. XXI. Instituto Mexicano del Seguro Social, México
}

\section{RESUMEN}

El objetivo del estudio fue determinar la confiabilidad y validez del CREED (Skinner, 2003) para medir la percepción de la enfermedad de adolescentes mexicanos con diabetes mellitus tipo 1 (DM1). Se evaluaron 85 adolescentes con DM1, de 11 a 18 años, sin otra comorbilidad. El CRED es un instrumento auto aplicable tipo Likert de cinco puntos y consta de cinco subescalas. Se realizó su traducción y adaptación al contexto mexicano y se sometió a laboratorio cognitivo para determinar su inteligibilidad. Los resultados mostraron su confiabilidad con consistencias internas aceptables por subescala (alfa de Cronbach $=0,64$ a 0,83). La validez de constructo se determinó mediante la concordancia de la estructura factorial con los constructos teóricos. Los resultados del CRED se correlacionaron significativamente con sintomatología depresiva $(r=0.227, p=0.040)$, demostrando validez convergente. Por lo tanto, la versión del CRED traducida al español y adaptada transculturalmente es confiable y válida.

Descriptores: diabetes tipo 1, adolescente, percepción de enfermedad, validez y confiabilidad, depresión.

\section{ABSTRACT}

The aim of the study was assess the reliability and validity of the DIRQ (Skinner, 2003) in Mexican adolescents with type 1 diabetes mellitus (T1DM) for measuring how they perceive their illness. The sample was 85 adolescents aged 11 to 18 with TIDM without comorbidities. The DIRQ is a self-report 5-point Likert scale comprised of 5 subscales. The questionnaire was translated and adapted to the Mexican context with the cognitive laboratory technique. It's reliability and validity were evaluated. Construct validity was assessed with factor analysis and convergent validity via the scales correlation with depressive symptoms. The internal consistency (Cronbach's alpha) for each subscale was acceptable (0.64-0.83). The factor structure was consistent with the theory. Illness perception correlated significantly with depressive symptoms $(r=0.227 p=0.040)$. DIRQ version translated into Spanish and adapted to the Mexican context, is a reliable and valid instrument to measure the perception of illness in Mexican adolescents with T1DM.

Keywords: Type 1 Diabetes, adolescent, illness perception, validity and reliability, depression.

\footnotetext{
* Para correspondencia: Dewi Sharon Montoya. Departamento de Salud Pública, 6to. Piso, Edificio B, Facultad de Medicina, Ciudad Universitaria, unaM, México, tel: (55)2753-7820, e-mail: dewishm@comunidad.unam.mx. Corina Benjet, Calzada México Xochimilco 101 San Lorenzo Huipulco, Tlalpan, 14370, Ciudad de México, tel:(55) 4160 5000, e-mail: cbenjet@imp.edu.mx. Elisa Nishimura Meguro. Av. Cuauhtémoc \#330, col. Doctores Ciudad de México, C. P. 06720, tel: (55)5627-6900, ext. 22292, e-mail: elisa.nishimura@imss.gob.mx. María Elena Hernández Méndez. Av. Cuauhtémoc \#330, col. Doctores, Ciudad de México, C. P. 06720, tel: (55)5627-6900, ext. 22292, e-mail: mehm@hotmail.com.
} 
La diabetes mellitus tipo 1 (DM1) es una de las enfermedades endocrinas más frecuentes entre los adolescentes mexicanos. Implica el alza de los niveles de glucosa en la sangre debido a la deficiencia total de insulina y a la imposibilidad de utilización de los macronutrientes. Para conseguir mantener estos niveles en parámetros aceptables es necesario tener un estricto apego terapéutico. Las demandas del tratamiento consisten en llevar una dieta estricta y calcular la cantidad de carbohidratos, así como en tomar las dosis necesarias de insulina. Asimismo, se requieren varias aplicaciones de insulina durante el día o tener un infusor y monitorear constantemente los niveles de glucosa y registrarlos para el ajuste de los requerimientos de ésta. Además, es necesaria la práctica de ejercicio físico regular (Pérez-Pastén \& Barrón, 1998; Searle, 2007).

A pesar de un apego estricto al tratamiento, no siempre es posible mantener los niveles de glucosa dentro de los ideales y pueden presentarse complicaciones que requieren hospitalización y ponen en riesgo la vida del adolescente. A largo plazo la diabetes puede causar complicaciones crónicas que comprometen la agudeza visual, la función renal y la sensibilidad de la persona con el diagnóstico, generándole hospitalizaciones, discapacidad, dependencia y muerte (Pérez-Pastén \& y Barrón, 1998; Kyngäs, 2000; Ortiz, 2004; MartínezChamorro, Lastra-Martínez Luzuriaga-Tomás, 2002; Storch, Heidgerken, Geffken, Lewin, Ohleyer, Freddo, et al., 2000).

La adolescencia es una etapa del ciclo vital en la que se produce la transición de la infancia a la adultez. Una enfermedad crónica como la diabetes mellitus tipo 1 puede interferir de forma negativa en el desarrollo del adolescente, generando alteraciones en la conducta que pueden condicionar un incumplimiento terapéutico y por ende, descontrol glucémico. Además, la demanda de una serie de conductas terapéuticas y hábitos estrictos de cuidado a la salud que pueden representar una fuente de estrés en el menor. Este estrés de forma crónica puede llevar al desarrollo de alteraciones en la salud mental el y estado afectivo de este grupo etario (Kyngäs, 2000; De la Fuente, 1992; Martínez-Chamorro et al. 2002).

La percepción de la enfermedad hace referencia a la atención, respuesta afectiva, cognición y valoración directa de la misma y sus manifestaciones. Dicha per- cepción se encuentra centrada directa y explícitamente en los aspectos subjetivos y significados que un enfermo le otorga a la experiencia de vivir con su padecimiento, y concluye con la construcción de una representación interna personal (Conrad, 1987; Weinman Petrie, 1997).

El individuo enfermo se confronta con un mal estado de salud que lo motiva, mediante respuestas emocionales y conductuales, a adoptar estrategias para afrontar su enfermedad. Las representaciones de la enfermedad determinan la implementación de estrategias de afrontamiento e implícitamente las respuestas emocionales. Después, estas respuestas son evaluadas por el mismo sujeto para determinar su efectividad, y en caso contrario, implementará otras alternativas más apropiadas. Este proceso de autorregulación se repite continuamente hasta que, en términos emocionales y conductuales, las estrategias resulten óptimas para lograr un estado de equilibrio con la enfermedad (Edgar \& y Skinner, 2003; Leventhal, Brissette \& Leventhal, 2003). La continuidad del proceso y reconstrucción mental adaptativa se estabilizan a largo plazo y permiten el ajuste con la enfermedad, que de resultar deficiente se torna patológico y susceptible de intervención (Bazzazian \& Besharat, 2012).

El modelo de autorregulación propuesto por Leventhal en 1980 explica la variación del curso de la enfermedad y de las conductas adaptativas resultantes de la integración de las creencias acerca de ésta en un grupo de individuos con un padecimiento en común (Law, Kelly, Huey \& Summerbell, 2002). Este modelo surge de la construcción de la representación propia de la enfermedad. Quien la padece percibe los problemas que involucran su papel de enfermo. Estas representaciones están compuestas por las percepciones de cinco componentes (Edgar \& Skinner, 2003; Leventhal et al., 2003; Searle, 2007; Weinman \& Petrie, 1997).

El primer componente causa se refiere a creencias sobre las causas de la enfermedad. El componente identidad involucra los síntomas y, en su caso, la etiqueta del padecimiento y la del paciente. El componente consecuencias considera el impacto psicológico y la amenaza percibida de las consecuencias que trae para el enfermo su padecimiento. Evolución contempla la percepción de cronicidad de la enfermedad. Finalmente, el componente control/curación es la va- 
loración subjetiva de control sobre la enfermedad y las consecuencias bajo un régimen terapéutico, o en su caso, la expectativa de curación (Edgar \& Skinner, 2003; Leventhal et al., 2003; Searle, 2007; Weinman \& Petrie, 1997).

La respuesta emocional de los adolescentes es resultado de su percepción. Se ha tenido evidencia de que el padecimiento de patologías crónicas se encuentra asociado a trastornos depresivos. Múltiples estudios han mostrado que adolescentes con diabetes tipo 1 presentan cuadros depresivos más prolongados, con peor pronóstico y con tasas de recurrencia más altas (Dantzer, Swendsen, Maurice-Tison \& Salamon, 2003; Grey, Cameron, Lipman \& Thurber, 1995; Kovacs, Goldston, Obrosky \& Bonar, 1997; Storch et al., 2006). La percepción de la enfermedad es un aspecto psicológico del enfermo que juega un papel importante en el establecimiento de conductas de cuidado a la salud $y$ en el bienestar emocional, que influyen de manera determinante en la evolución y pronóstico de la misma (Petrie, Jago \& Devchic, 2007; Haller, Sanci, Sawyer \& Patton, 2008). Resulta de suma importancia obtener conocimiento objetivo de la percepción de la diabetes, por lo cual es necesario un instrumento que pueda ofrecer dicha información.

Con base en el Modelo de Autorregulación de Leventhal se construyó el instrumento Illness Perception Questionnaire (IPQ) (Weinman \& Petrie, 1996). Más tarde se realizaron adaptaciones para múltiples enfermedades. Al respecto de la DM1 se construyó el Diabetes Illness Representation Questionnaire (DIRQ) (Skinner, Howells, Greene, Edgar, McEvilly \& Johansson, 2003), instrumento que fue diseñado para su aplicación en adolescentes con DM1 en el Reino Unido (Edgar \& y Skinner, 2003; Weinman \& y Petrie, 1996).

Los criterios para la adecuación de DIRQ involucraron la integración de ítems sobre la percepción de consecuencias del IPQ (Weinman \& Petrie, 1996), con algunos referentes a modelos personales de la diabetes de la Escala de Consecuencias y Efectividad del Tratamiento para evaluar la percepción de control de la enfermedad y prevención de sus complicaciones (Skinner et al., 2003).

El presente estudio detalla el trabajo mediante el cual el DIRQ (o CRED, por sus siglas en español) fue traducido y adaptado transculturalmente para poder desarrollar el proceso de validación del instrumento en la población mexicana. Lo anterior con el objetivo de contar con las herramientas necesarias para su posterior aplicación en el desarrollo de investigación acerca de la percepción de la enfermedad en nuestra población.

\section{MÉTODO}

\section{Participantes}

La muestra estuvo conformada por 85 adolescentes mexicanos con el diagnóstico de DM1 quienes aceptaron participar en el estudio bajo el consentimiento informado de su padre o tutor y el propio asentimiento del adolescente. Sus edades se situaron entre los 11 y 18 años, con una media de $14 \pm 2$ años, y el $60 \%$ fueron mujeres. El 74\% pertenecen a un nivel socioeconómico medio; $14.1 \%$ alto y $9.4 \%$ bajo. El $100 \%$ estudian, y de éstos, $76 \%$ tiene una escolaridad acorde con la edad. En cuanto a sus características clínicas, $80.5 \%$ de los adolescentes reciben atención médica en el Hospital de Pediatría del Centro Médico Nacional Siglo XXI y $13.4 \%$ ha sido egresados del mismo hospital. La edad de diagnóstico promedio es de $8 \pm 3$ años de edad y se reportó $6 \pm 3$ años de evolución de la enfermedad. El $80.3 \%$ de ellos siguen diariamente un esquema de insulina intensificado (tres o más aplicaciones) y 19.8\% manejan un esquema convencional (una o dos aplicaciones). El 53\% de los adolescentes se practica pruebas de glucosa diarias. El valor promedio de la última hemoglobina glucosilada (HbA1c) fue de $8.6 \%$ con una desviación estándar de $1.7 \%$.

\section{Instrumento}

El CRED es un instrumento auto aplicable tipo Likert de cinco puntos que van de totalmente de acuerdo a totalmente en desacuerdo, y de: nada importante a súper importante. Consta de cinco subescalas. La subescala de causa pregunta qué tan de acuerdo están con 13 posibles causas de la diabetes. La de consecuencias de la enfermedad, el impacto de éstas y la amenaza que representa la enfermedad, mediante seis reactivos. La sub escala de evolución pregunta sobre la percepción de cronicidad y cura de la diabetes mediante cuatro reactivos. En cuanto al control sobre la enfermedad y la prevención de complicaciones, se cuestiona qué 
tan importantes son los diferentes aspectos de su tratamiento para el control de la diabetes y para prevenir complicaciones utilizando 17 reactivos. Finalmente, la sub escala de identidad pregunta qué tanto el padecimiento afecta la vida cotidiana con seis reactivos. Puntuaciones altas en las sub escalas de identidad, evolución y consecuencias se asocian con una percepción negativa de la enfermedad. Así, puntuaciones altas para percepción de la efectividad del tratamiento se asocian con una percepción positiva de la enfermedad.

La sintomatología depresiva se midió con la versión en español para adolescentes del Inventario para Depresión de Beck (BDI) (Jurado, Villegas, Méndez, Rodríguez, Loperena y Varela, 1998). Se trata de un instrumento autoaplicable que consta de 21 ítems tipo Likert de cinco puntos que evalúan la severidad de los síntomas clínicos depresivos según el valor de cada una de las opciones de respuestas. Se le han asignado puntuaciones medias por severidad, discriminando entre sintomatología depresiva ausente o mínima, leve, moderada o grave.

\section{Procedimiento}

El procedimiento se desarrolló en dos fases. Primero se realizaron las técnicas de traducción y retraducción de la versión original en inglés, así como la adaptación por parte de especialistas del área y con lengua materna inglesa. La versión adaptada se sometió a la técnica de laboratorio cognitivo usando sondeo concurrente y espontáneo. La segunda fase consistió en la aplicación del CRED en la versión resultante y el Inventario para Depresión de Beck (BDI). Finalmente se evaluó la validez y confiabilidad del instrumento definitivo.

\section{Análisis estadístico}

El análisis estadístico se realizó con la versión 15 del paquete estadístico Statistical Package for the Social Sciences. Se realizó un análisis factorial exploratorio; se calculó el alfa de Cronbach para cada subescala y por total, así como el coeficiente de Pearson para relación entre la percepción de enfermedad y la sintomatología depresiva.

\section{RESULTADOS}

\section{Traducción y adaptación transcultural}

Se realizó la traducción y retraducción de la versión original del DIRQ por expertos en DM1 para la traducción y en investigación en niños y adolescentes, cuya lengua materna es el inglés, para la retraducción. Las adaptaciones realizadas surgieron de propuestas por parte de expertos en la práctica clínica con estos menores, y las adaptaciones finales y versiones definitivas con base en la experiencia con la población adolescente.

\section{Laboratorio cognitivo}

Existieron reactivos cuya traducción tenía un alto grado de dificultad para su comprensión, por lo cual, se realizaron modificaciones con base en propuestas hechas por los mismos adolescentes. Se eliminaron conceptos que resultaban confusos o incomprensibles en especial para los chicos en edades tempranas, y se sustituyó sustituyeron por un lenguaje con el cual el adolescente se identificara, pudiera otorgarle significado y apropiarse de los reactivos.

\section{Validez de constructo}

Para determinar la validez de constructo los reactivos se sometieron a análisis factorial exploratorio. Se realizó el cálculo de las matrices factoriales del instrumento con el método de extracción de componentes principales y rotación ortogonal por el método Varimax. La estructura factorial del instrumento fue calculada de forma fraccionada, tomando en cuenta los reactivos con las mismas opciones de respuesta.

Las subescalas con opción de respuesta totalmente de acuerdo-totalmente en desacuerdo son evolución, identidad y consecuencias. Debido a que esta última se conforma de dos componentes: impacto y amenaza, se realizó el correspondiente sub análisis. La subescala con opción de respuesta importante-súper importante es tratamiento y se analizaron sus componentes de control y prevención. Los resultados de los reactivos de la versión definitiva, que son los que mostraron validez de constructo, se muestran en la tabla 1 . 
Tabla 1. Matrices factoriales de las subescalas evolución e identidad (A) y componentes de las subescalas consecuencias (B) y tratamiento (C)

\begin{tabular}{|c|c|c|c|c|c|}
\hline \multirow{2}{*}{$\begin{array}{c}\text { Reactivos } \\
\text { (A) }\end{array}$} & \multicolumn{2}{|c|}{ Carga factorial } & \multirow{2}{*}{$\begin{array}{c}\text { Reactivos } \\
\text { (B) }\end{array}$} & \multicolumn{2}{|c|}{ Carga factorial } \\
\hline & Evolución & Identidad & & Amenaza & Impacto \\
\hline Evolución 1 & .825 & & & & \\
\hline Evolución 2 & .786 & & Amenaza 1 & .736 & \\
\hline Evolución 3 & .837 & & Amenaza 2 & .751 & \\
\hline Evolución 4 & .797 & & Amenaza 3 & .672 & \\
\hline Identidad 1 & & .778 & Impacto 1 & & .803 \\
\hline Identidad 2 & & .578 & Impacto 2 & & .480 \\
\hline Identidad 3 & & .520 & Impacto 3 & & .697 \\
\hline Identidad 4 & & .719 & & & \\
\hline Identidad 5 & & .368 & & & \\
\hline Identidad 6 & & .550 & & & \\
\hline Reactivos & Carg & rial & Reactivos & Car & \\
\hline (C) & Prevención & Control & (C) & Prevención & Control \\
\hline Prevención 1 & .679 & & Control 1 & & .595 \\
\hline Prevención 3 & .890 & & Control 3 & & .511 \\
\hline Prevención 4 & .571 & & Control 4 & & .474 \\
\hline Prevención 7 & .920 & & Control 7 & & .041 \\
\hline Prevención 8 & .786 & & Control 8 & & .502 \\
\hline Prevención 9 & .856 & & & & \\
\hline
\end{tabular}

Varianza explicada por reactivos A y B 49. 3\% A.43.9\%, B 54.9\% C 52.9\%

En el análisis de la subescala de tratamiento se eliminaron los reactivos de ambos componentes que preguntan acerca de "hablar con otras personas sobre lo que siento por tener diabetes", "no fumar" y "no comer muchas cosas dulces" por no presentar validez de constructo. Es importante señalar que estos reactivos exploran la percepción sobre conductas de cuidados a la salud que la muestra no identificó como parte de sus conductas de auto cuidado para la enfermedad.

El análisis factorial realizado para los reactivos de la subescala de percepción de la causa tuvo la finalidad de agrupar las posibles causas planteadas en factores comunes. Los resultados finales del análisis para dos factores mostraron una matriz factorial con saturación del factor 1, con percepción de causas emocionales e ideas mágicas "por mala suerte", "por mi forma de ser", mientras el factor 2 es saturado por las creencias populares más frecuentes de la génesis de la diabetes: "por la herencia" y "por las cosas que como o comía”.

\section{Validez concurrente}

Para evaluar la validez concurrente del instrumento con el nivel de sintomatología depresiva se realizó el análisis de correlación lineal entre los puntajes del DIRQ y el BDI. Se encontró que los resultados correlacionan significativamente $(p=0.046)$ y muestran un coeficiente de correlación de Pearson $r=0.227$. 


\section{Confiabilidad}

La confiabilidad del instrumento se determinó por el análisis de consistencia interna mediante el cálculo del alfa de Cronbach. Se evaluaron las consistencias internas por subescala y global. Para el análisis fue necesario fraccionar de acuerdo con los reactivos que tuvieran las mismas opciones de respuesta, así, el grupo que obtuvo las mismas opciones de respuesta 1 incluye a las subescalas de evolución, sintomatología, consecuencias y causa, dejando al grupo que obtuvo las mismas opciones de respuesta 2 compuesto por la subescala de tratamiento en sus componentes de control y prevención.

Para analizar la consistencia interna global del instrumento se realizó el cálculo del alfa de Cronbach de acuerdo con el agrupamiento de las subescalas con las mismas opciones de respuesta. Para las subescalas de evolución, identidad, consecuencias y causa, el alfa calculada fue de 0.610. Finalmente, para la subescala de tratamiento el alfa de Cronbach resultante fue de 0.778 (ver tabla 2 ).

Tabla 2. Consistencia interna global y por subescalas según el tipo de opciones de respuesta

\begin{tabular}{|l|l|c|}
\hline Subescala & Componente & $\begin{array}{c}\text { Consistencia } \\
\text { (alfa) }\end{array}$ \\
\hline Evolución & & 0.830 \\
\hline Identidad & Total & 0.642 \\
\hline Consecuencias & Impacto & 0.354 \\
\hline Causa & Amenaza & 0.597 \\
\hline Consistencia total & $\begin{array}{l}\text { Mismas opciones } \\
\text { de respuesta 1 }\end{array}$ & 0.460 \\
\hline Tratamiento & Total & 0.792 \\
\hline & Control & 0.513 \\
\hline Consistencia total & $\begin{array}{l}\text { Mismas opciones } \\
\text { de respuesta 2 }\end{array}$ & 0.778 \\
\hline
\end{tabular}

\section{DISCUSIÓN}

El proceso de validación del instrumento en sus diferentes fases se vio guiado por la necesidad de adaptación, análisis e interpretación de los datos, con fundamento en las características de la muestra y el contexto mexicano. La versión traducida original incluía tecnicismos, que a pesar de ser comprensibles para los adolescentes, no permitían que lograran apropiarse del contenido para expresar su percepción sobre la enfermedad sino que dirigían sus respuestas al conocimiento de la educación en diabetes. La adaptación transcultural se dirigió a permitir al adolescente identificarse con lo que se le pregunta, evitar un tono de evaluación de conocimientos y lograr la sensación de confianza al ofrecer sus respuestas.

Por otro lado, la estructura factorial agrupó a los reactivos de forma congruente con lo propuesto en la teoría con respecto a los aspectos contemplados por el modelo de autorregulación. El análisis fue muy consistente con la disposición de los reactivos de las diferentes subescalas del DIRQ (Covo, 1973). Sin embargo, para nuestra población, la estructura factorial de la subescala de tratamiento no se mostró claramente definida para algunos aspectos clínicos, contemplados por el instrumento. Estos reactivos abordan la percepción sobre conductas de cuidados a la salud o su evitación. Estas conductas son hábitos saludables que los adolescentes perciben como importantes pero no como parte del autocuidado de la diabetes mellitus, sino como medidas de cuidado a la salud, no específicas para su padecimiento, evidenciando la carencia de validez de constructo de estos reactivos particulares con respecto a la teoría.

La consistencia interna de la versión traducida del DIRQ resultó muy similar a la reportada en los dos estudios realizados por el autor para la validación del instrumento original (Covo, 1973). El alfa obtenido para los componentes de las subescalas de consecuencias y tratamiento son consistencias bajas. Sin embargo, el número de reactivos analizados es muy reducido (3-4) para cada componente, motivo por el cual la consistencia interna puede encontrarse subestimada, debido a la disminución de la varianza y la baja capacidad de mostrar un espectro discriminante del componente (Covo, 1973). Al realizar el análisis por subescalas completas, 
es decir, estimando la consistencia de los reactivos de sus componentes, se obtienen valores adecuados.

La sintomatología depresiva es un constructo teórico reportado en la literatura como una variable asociada a la percepción de la enfermedad. El instrumento evalúa aspectos emocionales dirigiéndose a preguntar qué tanto los síntomas de la DM1 afectan la vida cotidiana del adolescente. Especialmente la subescala de identidad explora su autopercepción y la percepción de los otros hacia él por presentar estas manifestaciones, los problemas que derivan de las mismas, así como el impacto que tienen en la cotidianidad. Sin embargo, el instrumento en su totalidad no se dirige sólo a conocer estas percepciones, sino que lo hace en aspectos como la eficacia del tratamiento que están más a cogniciones. Esto puede explicar que la correlación obtenida con el nivel de sintomatología depresiva, aunque significativa, fuera baja (Martínez-Chamorro, Lastra-Martínez \& Luzuriaga-Tomás, 2002; Edgar \& Skinner, 2003; Leventhal, Brissette \& Leventhal, 2003).

Entre las limitaciones del estudio podemos mencionar que la muestra estuvo conformada en su mayor parte por adolescentes que siguen programas de educación en diabetes y reportan percepciones sesgadas por el antecedente de la educación. Esto fue claramente observable al responder preguntas que involucran el conocimiento sobre algunos aspectos clínicos de la enfermedad, tales como los de la subescala de tratamiento. Por lo cual se puede concluir que la muestra no resulta representativa de la población de adolescentes mexicanos con diabetes mellitus tipo 1, y por ende

\section{REFERENCIAS}

Bazzazian, S. \& Besharat, M. A. (2012). An explanatory model of adjustment to type I diabetes based on attachment, coping, and self-regulation theories. Psychology \& Health Medicine, 17(1), 47-58.

Conrad, P. (1987). The experience of illness: recent and new directions. En P. Conrad y J. Roth. The experience and management of chronic illness. Research in the Sociology of Health Care, 6, 1-31.

Covo, M. (1973). Conceptos comunes en la metodología de la investigación sociológica. En M. Covo (Ed.), los resultados obtenidos no pueden ser generalizados a todo ese universo.

El instrumento explora aspectos clínicos de la enfermedad que abordan la educación en diabetes. Las respuestas de los adolescentes podrían verse sesgadas por la deseabilidad social, ya que quizá reporten percepciones acordes con la educación que reciben y no necesariamente con los sentimientos y pensamientos propios. Esto también se pudo ver reforzado por el ámbito hospitalario en el cual se llevó a cabo.

A pesar de estas limitaciones, podemos concluir que el Cuestionario de representaciones de la enfermedad para diabetes en su versión traducida al español muestra una confiabilidad y validez adecuada para medir la percepción de la enfermedad de los adolescentes con diabetes mellitus tipo 1 mexicanos, y por ende proporciona una herramienta útil para detectar e intervenir en problemas relacionados con el apego al tratamiento o las respuestas emocionales negativas que surgen como consecuencia de tener que lidiar con esta enfermedad crónica. Sin embargo, resulta incompleto para obtener un acercamiento más profundo a los motivantes culturales que inciden en las percepciones de estos adolescentes, en relación con las características de la cultural mexicana. La evaluación de la percepción de la enfermedad, involucrando aspectos culturales como creencias, mitos y el estigma, resultaría complementaria para profundizar en la construcción de la representación de la diabetes de estos adolescentes y las respuestas emocionales que les genera en favor de su salud mental.

Manual para estudiantes (137-152). México: UnAM. Instituto de Investigaciones Sociales.

Dantzer, C., Swendsen, J., Maurice-Tison, S. \& Salamon, R. (2003). Anxiety and depression in juvenile diabetes: a critical review. Clinical Psychology Review, 23(6), 787-800.

De la Fuente, R. (1992). El ciclo vital. En R. De la Fuente (Ed.), Psicología médica (91-53), México: Fondo de Cultura Económica. 
Edgar, K. \& Skinner, T. C. (2003). Illness representations and coping as predictors of emotional well-being in adolescents with type 1 diabetes. Journal of Pediatric Psychology, 28(7), 485-493.

Grey, M., Cameron, M., Lipman, T. \& Thurber, F. (1995). Psychosocial status of children with diabetes in the first 2 years after diagnosis. Diabetes Care, 18, 13301336.

Haller, D. M., Sanci, L. A., Sawyer, S. M. \& Paton, G. (2008). Do young people's illness beliefs affect healthcare? A systematic review. Journal of Adolescent Health, 42, 436-449.

Jurado, S., Villegas, M., Méndez, L., Rodríguez, F., Loperena, V. \& Varela, R. (1998). La estadarización del Inventario de Depresión de Beck para los residentes de la Ciudad de México. Salud Mental, 24(3), 26-31.

Kovacs, M., Goldston, D., Obrosky, D. \& Bonar, L. (1997). Major depressive disorder in youths with IDDM. Diabetes Care, 20, 45-50.

Kyngäs, H. (2000). Compliance of adolescents with chronic disease. Journal Clinical Nursing, 9(4), 549-56.

Law, G., Kelly, T., Huey, S. \& Summerbell, C. (2002). SelfManagement and well-being in adolescents with diabetes mellitus: Do illness representations play a regulatory role? Journal of adolescent health, 31(1), 981-385.

Leventhal, H., Brissette, I. \& Leventhal, E. (2003). Conceptualizing self-regulation as regulation of the self system. En H. Leventhal \& L. Cameron (Eds.), The regulation of health and illness behavior (42-96). Reino Unido: Routledge.

Martínez Chamorro, H. J., Lastra-Martínez, I. \& Luzuriaga Tomás, C. (2002). Perfil psicosocial de niños y adolescentes con diabetes mellitus. Boletín de la Sociedad de Pediatría de Asturias, Catambria, Castilla y León, 42, 114-119.
Ortiz, M. (2004). Factores Psicológicos y Sociales Asociados a la Adherencia al Tratamiento en Adolescentes Diabéticos Tipo 1. Psykhe, 1, 21-31.

Pérez-Pastén, E. \& Barrón, C. (1998). Diabetes Mellitus tipo 1. En J. Games-Eternod \& J. Palacios (Eds.). Introducción a la pediatría (pp. 967). México: Méndez Editores.

Petrie, K. J., Jago, L. A. \& Devcich, D. A. (2007). The role of illness perceptions in patients with medical conditions. Current Opinion in Psychiatry, 20(2), 163-167.

Searle, A. (2007). Illness representations among patients whith type 2 diabetes and their partners: relationships with self-management behaviors. Journal Phsychosomatic Research, 63, 175-184.

Skinner, T. C., Howells, L., Greene, S., Edgar, K., McEvilly, A. \& Johansson, A. (2003). Development, reliability and validity of the Diabetes Illness Representations Questionnaire: four studies with adolescents. Diabetic Medicine, 20(4), 283-289.

Storch, E. A., Heidgerken, A. D., Geffken, G. R., Lewin, A. B., Ohleyer, V., Freddo, M. \& Silverstein, J. H. (2006). Bullying, regimen self-management, and metabolic control in youth with type I diabetes. The Journal of Pediatrics, 148(6), 784-7877.

Weinman, J. \& Petrie, K. (1996).The Illness Perception Questionnaire: A New Method for Assessing the Cognitive Representation of Illness. Psychol Health, 11, 431-435.

Weinman, J. \& Petrie, K. (1997). Illness perceptions: a new paradigm for psychosomatics? Journal of Pshychosomatic Research, 42(2), 113-116.

NotA DE LOS AUTORES: Agradecemos la contribución del Dr. T. Chas Skinner por su autorización y apoyo para la utilización de la versión original del instrumento DIRQ. 